\title{
Field Application of a Combined Vaccine against Peste Des Petits Ruminants and Sheep Pox
}

\author{
Fakri $\mathbf{F}^{1,2^{*}}$, Ghzal $\mathbf{F}^{1}$, Daouam $\mathbf{S}^{1}$, Elarkam $\mathbf{A}^{1}$, Douieb $L^{1}$, Tadlaoui $\mathbf{K}^{1}$, Fassi-Fihri $\mathbf{O}^{2}$ and Elharrak $\mathbf{M}^{1}$
}

${ }^{1}$ Research and Development, Multi-Chemical Industry, Lot. 157, Z I, Sud-Ouest (ERAC) B.P: 278, Mohammedia 28810, Morocco

${ }^{2}$ Institut Agronomique et Vétérinaire Hassan II, Rabat, Morocco

*Corresponding author: Fakri F, Multi-Chemical Industry, Lot. 157, Z I, Sud-Ouest (ERAC) B.P: 278, Mohammedia, Morocco; Tel.: +212670936062; E-mail: fz.fakri@mci-santeanimale.com

Received date: Oct 26, 2015; Accepted date: Nov 20, 2015; Published date: Nov 25, 2015

Copyright: (c) 2015 Fakri F, et al. This is an open-access article distributed under the terms of the Creative Commons Attribution License, which permits unrestricted use, distribution, and reproduction in any medium, provided the original author and source are credited.

\begin{abstract}
A combined vaccine against peste des petits ruminants (PPR) and sheep/goat pox (SGP), based on Nigeria 75 PPR strain and sheep pox Romania strain, was developed and applied in the field. Safety and efficacy were evaluated on goats and sheep in comparison with monovalent PPR and SGP vaccines. Goats were challenged by PPR virulent strain and sheep by SP virulent strain. The result showsthat the combined PPR/SGP vaccine confers a good protection against both PPR and SGP infection with no significant difference with monovalent vaccines. The combined vaccine was used in Morocco and other African countries on small ruminant flocks with good safety and satisfactory sero-conversion detected against both PPR and SGP viruses, as soon as 14 days post vaccination. It's the first time where a combined vaccine was applied in large vaccination campaigns to prevent in one shot the two diseases.
\end{abstract}

Keywords: Peste des petits ruminants; Sheep pox; Combined vaccine.

\section{Introduction}

Peste des petits ruminants (PPR) and sheep/goat pox (SGP) are highly contagious viral diseases of small ruminants causing huge economic losses. Both diseases are found in the same geographic areas. Vaccination using monovalent live vaccines is normally applied, however, the low vaccination coverage due to large space distribution of the animal population and poor infrastructure with difficult access contribute to spread or maintain the infection.

The control of PPR and SGP is a major goal for a program aimed to poverty alleviation because of the high importance of sheep and goats in endemic regions. A combined vaccine that protects against both infections in one shot may promote a wider use of vaccination. The benefits are numerous: to provide comfort to farmers, reduce stress in animals and minimize vaccination costs. A similar combination has been developed in the past and used experimentally with satisfactory results in India [1,2] and in Cameroon [3]. However, no mass vaccination has been conducted with the combined vaccine so far.

\section{Materiel and Methods}

The developed combined vaccine was based on highly immunogenic worldwide used strains of PPR (Nigeria 75) [4] and SP (Romania) [5]. Both strains grown on monkey african green kidney (VERO) cells. This strain association was tested for the first time

Animal experiment was carried out in accordance with guidelines for care and handling of experimental animals, as per the laboratory committee for purpose of control and supervision of experiments on animals. The experiment was conducted on four groups of animals housed in the animal unit of MCI Santé Animale, Mohammedia,
Morocco: Group 1 composed by 6 sheep and 6 goats vaccinated with the combined SGP/PPR, Group 2 composed by 4 sheep vaccinated with the monovalent SP, Group 3 composed by 4 goats vaccinated with the monovalent PPR and Group 4 composed by unvaccinated animals ( 2 goats and 2 sheep). Common local sheep breed and Alpine goats [6] were used in this experiment. All animals were aged 6-8 months, and tested sero-negative for PPR and SGP.

Vaccination of each group was conducted by subcutaneous route and the monitoring consisted on a clinical observation, temperature, injection site inflammation and serological response [7]. Determination of the vaccine potency was carried out by challenge on BSL3 containment laboratory. Goats and sheep were challenged at day (D) 28 pv. Goats were challenged by PPRV virulent strain according to the protocol of Elharrak [8] and sheep by SP virulent strain using the protection index protocol [9]. The obtained titre for the vaccinated group was compared with the titre of the unvaccinated control animals and the difference between the two titres expressed in log represent the protection index [9]. The monitoring consisted on daily observations of specific symptoms, temperature and local inflammation on the injection site. Clinical scoring for PPR and protection index for SP was determined for each animal and the average for the group was calculated [7]. All surviving animals were euthanized at D14 post infection, autopsied and sampled for further investigations.

The combined vaccine was then tested in the normal conditions of the field in three farms representing three breeding regions in Morocco [7]. To monitor vaccination response for both PPR and SGP, serological testing has been done using virus neutralization test $(\mathrm{SN})$ as described in the OIE Terrestrial Manual (Chapters 2.7.11\&2.7.14). ELISA test was also used to detect PPR kinetic of antibodies following vaccination. The kit 'ID Screen PPR Competition reference (PPRC-4P ID-VET) was used for that purpose [10]. 
For the antigen detection on challenged animals, we used real time qPCR as described by Batten [11] for PPR and by Bowden [12] for SGP. DNA extraction was performed using isolate genomic DNA/RNA Mini kit (Bioline BIO-52066 \& BIO-52075) and amplification done with the Kit superscript Tm III Platinum R one step qRt-PCR system (Cat. no. 11745-100).

\section{Results and Discussion}

In this experiment, a monovalent PPR vaccine, Nigeria 75 strain, a monovalent SGP vaccine, Romania strain and a combined PPR/SGP vaccine, on lyophilized forms were produced. The infectious titres per dose for these vaccines were 104.1 TCID50 for PPR, and 104.0 TCID50 for SGP [7].

Safety and efficacy of the vaccination was evaluated on animals comparatively between combined and monovalent vaccines. During the three weeks following vaccination, all vaccinated animals remained healthy, without any effect on their appetite and behaviour. No abnormal local reactions have been reported, except of a small transitory nodules and a short increase of the temperature not exceeding $1^{\circ} \mathrm{C}$ during 2 days in sheep of Group 1 and Group 2. This reaction is routinely observed with Sheep pox live attenuated vaccine based on Romania strain [5].

Serological response after vaccination is reported in figures 1 and 2. Good SGP antibody response obtained as soon as 7 days post vaccination. The neutralisation titre seems to stabilize between 1.8 and $2 \log$ (Figure 2).

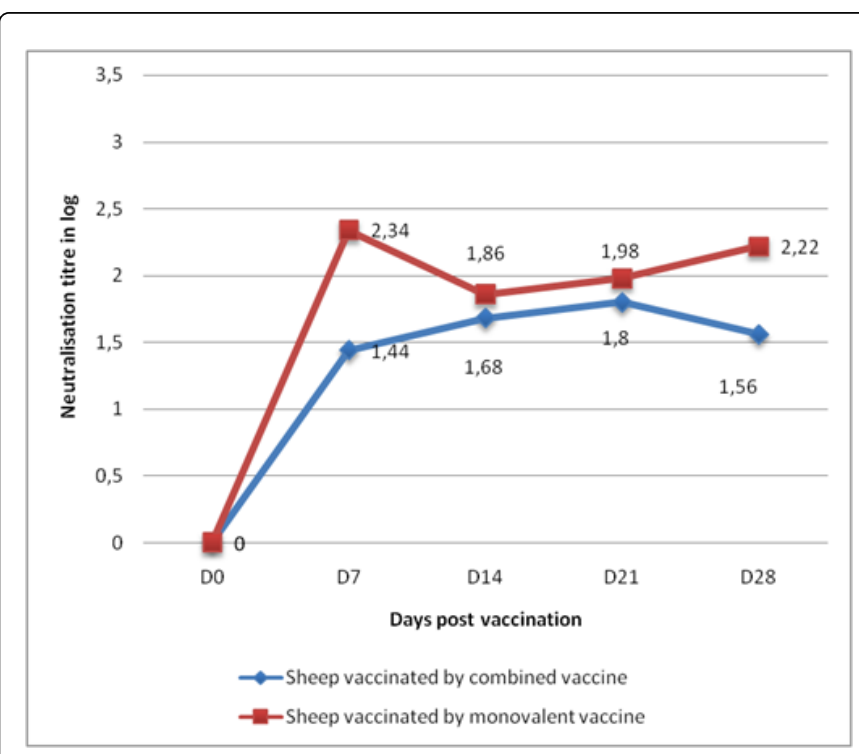

Figure 2: Neutralising SPV antibody response (in $\log 10$ ) after vaccination of sheep by combined and monovalent vaccines (group average).

Regarding PPR, the antibody titre increased progressively in goats until D28 with no significant difference ( $p>0.05)$ between combined or monovalent vaccine as tested by SN (Figure 1) and confirmed by ELISA. For sheep a similar response was obtained with PPR antibodies after vaccination (Figure 1). No significant difference detected in serological response between monovalent and combined vaccine or between sheep and goats regarding PPR valence. A similar observation was reported by Ayalet [13] and by Chaudhary [1].

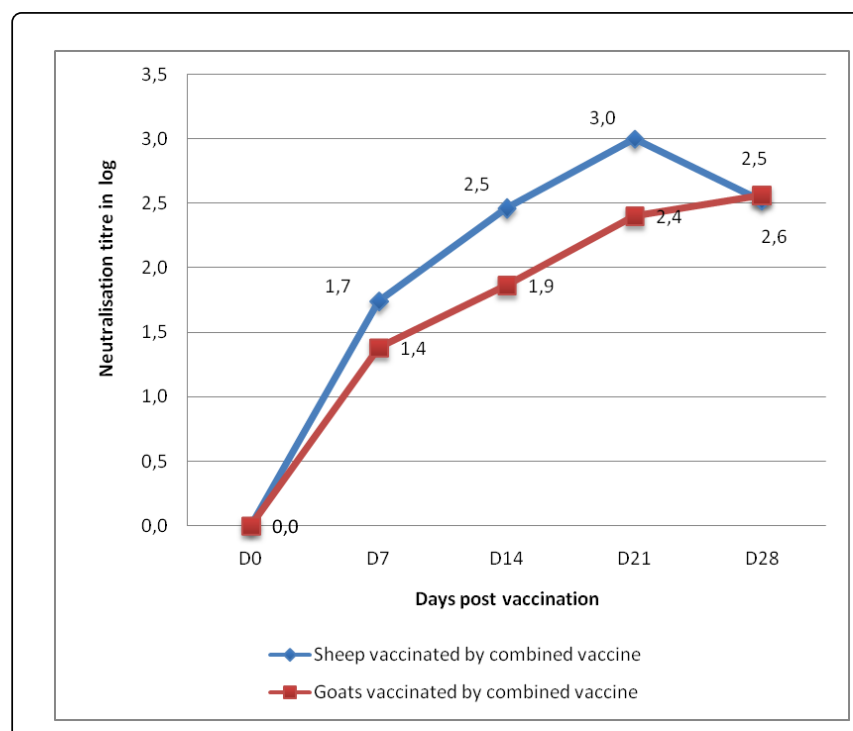

Figure 1: Neutralizing PPR antibody response (in $\log 10$ ) after vaccination of sheep and goats by combined vaccines (group average).

Regarding SGP, serological response tested in sheep after vaccination show no significant difference between monovalent and combined vaccine. This valence has not been tested on goats because it has been demonstrated previously that Romania strain provides good protection against Goat Pox infection: vaccinated goats challenged with a virulent strain of Goat Pox virus appears to be fully protected (unpublished data). This cross protection between genus of Capripox viruses have been reported by several authors $[14,15]$.

Both vaccinated and unvaccinated control animals were challenged 28 days after vaccination by the corresponding virulent strains. PPR challenge was conducted on goats [7]. Unvaccinated animals showed specific clinical signs of PPR infection: dyspnoea, nasal and ocular discharge followed by a respiratory syndrome with painful polypnea and profuse diarrhoea in the terminal stage. The body temperature exceeded $40.8^{\circ} \mathrm{C}$. The two unvaccinated control goats died at D8 and D10 post infection with a clinical scoring of 19 and 17 respectively. Analysis by qPCR showed a high level of viral excretion detected in ocular and rectal swabs (Ct 14.7). At post mortem examination, specific lesions of pneumonia and inflammatory nodules were observed in lung and digestive tract with high viral concentration as detected by qPCR (Ct 17.9 in mesenteric nodes and 19.4 in lungs).

The obtained results complain with other studies on alpine goats reported by Hammouchi et al., [16] and Elharrak et al., [8] who defined a challenge model for PPR. However other experimental infection carried out by several authors' shows irregular or nonconclusive results depending on the animal species and breeds, the challenge strain and inoculated doses [17]. For vaccinated animals with monovalent PPR or combined vaccine, no clinical symptom was reported during the 14 days of observation period, the body temperature remained normal and the clinical scoring estimated between 0 and 2. PCR testing of ocular and rectal swabs of vaccinated and challenged animals confirmed the absence of virus excretion. 
These give evidence that combined and monovalent vaccines provide complete protection against the disease and the infection with PPRV.

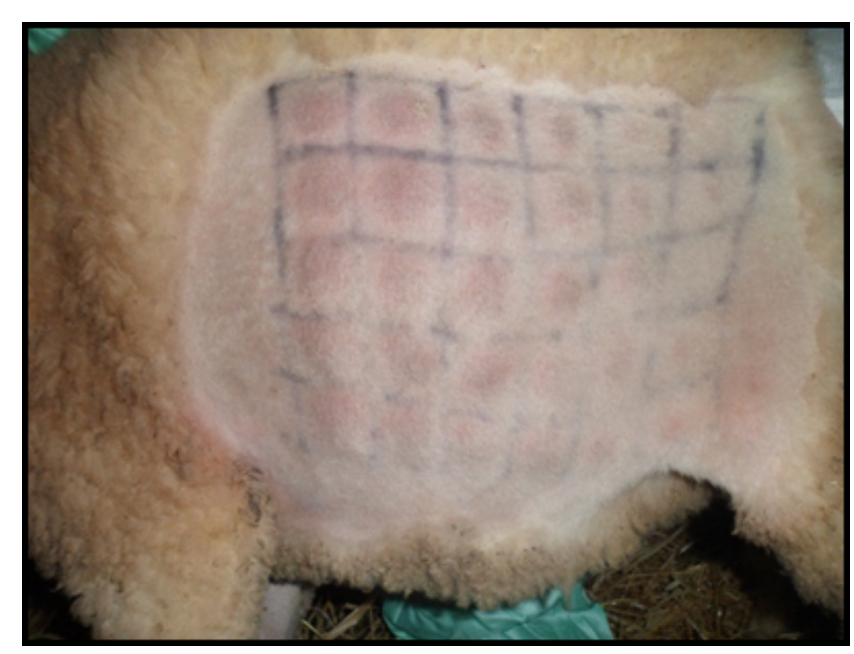

Figure 3: Picture of a challenged control sheep showing local inflammation on site of inoculation (flank) with $10-1$ to $10-6$ dilutions (left to right) of virulent SGPV in five replica.

SGP challenge was conducted on sheep [7]. The used challenge model allows quantification of the conferred immunity and then comparison between different vaccines. After challenge, unvaccinated sheep showed local inflammation in the site of injection (Figure 3) starting at D3 pi at the lowest dilution with the maximum reaction observed between D7 and D9. The two control animals presented hyperthermia with a peak value up to $41.4^{\circ} \mathrm{C}$ at $\mathrm{D} 7 \mathrm{pi}$, and secondary lesions fromD8 pi. The obtained titre was 5.5 and $5.9 \mathrm{ID} 50 / 0.2 \mathrm{ml}$ in the two controls respectively. After euthanasia at D14 pi, qPCR analysis showed a maximum viral charge with a Ct 11.3 in primary lesions (inoculation site), Ct 12.9 in secondary lesions (skin papules); an important viral charge was also observed in some nodes (Ct 9.72 in mesenteric nodes, to 17.2 in pulmonary node) and a low charge in lung (Ct 27.2) and negative in spleen.

Regarding vaccinated sheep, no clinical sign of the disease was observed and no secondary lesions reported for both monovalent and combined vaccine. After the challenge virus injection in the animal flank, a hypersensitivity reaction was observed after the first and the second day pi which disappear completely the following days. No inflammatory reactions reported in the injection sites even in the lowest dilution giving evidence of the complete neutralisation of the virulent virus by the conferred immunity. Similar results have been also reported by Precausta et al., [5], whose authors demonstrated that Romania strain is very effective for protection against sheep pox infection. The protection index was evaluated to 5.7ID50/0.2 $\mathrm{ml}$ in vaccinated sheep which mean a solid and durable immunity. In support of the present observations, previous studies conducted by Ayalet et al., [13] and by Chaudhary et al., [1] showed that all the immunized animals resisted challenge with virulent SPV or PPRV, while control animals developed characteristic signs of diseases.

The combined vaccine was then tested at large scale under normal field conditions on three flocks of sheep in the central region of Morocco. Results were expressed by percentage of sero-positive animals as tested by SN for PPR and SGP (Table 1). In two farms, few animals were found positive for PPR due to a previous vaccination. The global serological response obtained by ELISA reaches almost $100 \%$ at D14 after vaccination with some differences between farms. When tested by SN, 75\% of the animals were protected at D14 and $100 \%$ at D21 against PPR (Table 1). This difference between the two technics could be explained by high sensitivity of ELISA compared to SN. For SGP $65 \%$ of animals were protected at D14 to around $90 \%$ at D21 pv (Table 1). Consistent results were published by Hosamani et al., [2] using combined PPR and goat pox vaccine and by Sreenivasa [18] using PPR vaccine only.

\begin{tabular}{|l|l|l|l|l|l|}
\hline \multicolumn{2}{|l|}{ Days post vaccination } & D0 & D7 & D14 & D21 \\
\hline \multirow{3}{*}{ PPR } & Farm 1 & 15 & 30 & 95 & 100 \\
\cline { 2 - 6 } & Farm 2 & 0 & 0 & 50 & 100 \\
\cline { 2 - 6 } & Farm 3 & 0 & 5 & 80 & 100 \\
\hline \multirow{2}{*}{ PPR average value } & 5 & 12 & 75 & 100 \\
\hline \multirow{2}{*}{ SPV } & Farm 1 & 10 & 45 & 70 & 85 \\
\cline { 2 - 6 } & Farm 2 & 0 & 10 & 55 & 90 \\
\cline { 2 - 6 } & Farm 3 & 0 & 5 & 70 & 92 \\
\hline \multirow{2}{*}{ SPV average value } & 3 & 20 & 65 & 89 \\
\hline
\end{tabular}

Table 1: Vaccination monitoring: percentage of positive animals after vaccination by the combined PPR/SGP vaccine in three farms representingthree breeding regions in Morocco.

Consequently, the combined vaccine was selected and used in mass vaccination campaign in some African countries, mainly Morocco and Uganda.

\section{Conclusion}

The combined PPR/SGP vaccine confers in one shot a good protection against both PPR and Sheep pox infections and diseases. At laboratory level solid protection was obtained with no viral multiplication in vaccinated and challenged animals, results confirmed by serology monitoring after large scale vaccination under normal field conditions. The combined vaccine could be used for vaccination campaigns to protect small ruminants in one shot against two economically and medically major diseases. The combined vaccine could be an efficient tool in endemic countries with low infrastructure and extensive breeding management of small ruminant population. In addition, it could be interesting to use this combined vaccine to reduce vaccination costs which might be highly attractive for farmers in developping countries.

\section{References}

1. Chaudhary SS, Pandey KD, Singh RP, Verma PC, Gupta PK (2009) A vero cell derived combined vaccine against sheep pox and Peste des Petits ruminants for sheep. Vaccine 27: 2548-2553.

2. M Hosamani, SK Singh, B Mondal, A Sen, V Bhanuprakash et al. (2006) A bivalent vaccine against goat pox and Peste des Petits ruminants induces protective immune response in goats. Vaccine 24: 6058-6064.

3. A Martrenchar, N Zoyem, A Diallo (1997) Experimental study of vaccine against Peste des Petits Ruminants and capripox infection in goats in northern Cameroon. Small Ruminant Research 26: 39-44. 
Citation: Fakri F, Ghzal F, Daouam S, Elarkam A, Douieb L, et al. (2015) Field Application of a Combined Vaccine against Peste Des Petits Ruminants and Sheep Pox. J Vaccines Vaccin 6: 301. doi: 10.4172/2157-7560.1000301

Page 4 of 4

4. Diallo A, Taylor WP, Lefèvre PC, Provost A (1989) [Attenuation of a strain of rinderpest virus: potential homologous live vaccine]. Rev Elev Med Vet Pays Trop 42: 311-319.

5. Précausta P, Kato F, Vellut G (1979) A new freeze-dried living virus vaccine against sheep-pox. Comp Immunol Microbiol Infect Dis 1 : 305-319.

6. A Hajjam (2015) Etude expérimentale de la sensibilité comparée des races ovines et caprines marocaines au virus de la Peste des Petits Ruminants. Veterinary thesis, Institut Agronomique et Vétérinaire Hassan II, Rabat Morocco.

7. F Fakri, F Ghzal, S Daouam, A Elarkam, L Douieb, et al. (2015) Development and field application of a new combined vaccine against Peste des Petits Ruminants and Sheep Pox. Trials in Vaccinology 4: 33-37.

8. M Elharrak, NTouil, C Loutfi, M Hammouchi, S Parida, (2012) A reliable and reproducible experimental challenge model for Peste des Petits Ruminants virus. Journal of Clinical Microbiology 50: 3738-3740.

9. M Fassi-Fehri, M E Harrak, D Johnson, M Abbadi, AH El-Idrissi (1984) Etude expérimentale de l'immunité anti-claveleuse post-vaccinale. Annales de Recherches Vétérinaires 15: 59-64.

10. G Libeau, C Préhaud, R Lancelot, F Colas, L Guerre (1995) Development of a competitive ELISA for detecting antibodies to the Peste des Petits Ruminants virus using a recombinant nucleoprotein. Res Vet Sci 58: 50-55.

11. Batten CA, Banyard AC, King DP, Henstock MR, Edwards L, et al. (2011) A real time RT-PCR assay for the specific detection of Peste des petits ruminants virus. J Virol Methods 171: 401-404.
12. Bowden TR, Babiuk SL, Parkyn GR, Copps JS, Boyle DB (2008) Capripoxvirus tissue tropism and shedding: A quantitative study in experimentally infected sheep and goats. Virology 371: 380-393.

13. G Ayalet, N Fasil, S Jembere, G Mekonen, TSH Negussie (2012) Study on immunogenicity of combined sheep and goat pox and Peste des Petits Ruminants vaccines in small ruminants in Ethiopia. African Journal of Microbiology Research 6: 7212-7217.

14. RP Kitching (1986) The control of sheep and goat pox. Rev Sci Tech Off Int. Epizoot 5: 503-511.

15. A Diallo, JV Gerrit (2007) Genus Capripoxvirus, Poxviruses. Birkhäuser. Advances in Infectious Diseases, pp. 167-181.

16. Hammouchi M, Loutfi C, Sebbar G, Touil N, Chaffai N, et al. (2012) Experimental infection of alpine goats with a Moroccan strain of peste des petits ruminants virus (PPRV). Vet Microbiol 160: 240-244.

17. A Assim, A Rashid, AH Chaudhary, MS Noor (2009) Production of homologous live attenuated cell culture vaccine for the control of Peste des Petits Ruminants in small ruminants. J Pakistan Vet 29: 72-74.

18. BP Sreenivasa, P Dhar, RP Singh, SK Bandyopadhyay (2000) Evaluation of an indigenously developed homologous live attenuated cell culture vaccine against peste des petits ruminants infection of small ruminants. In: Proceedings of the XX annual conference of Indian association of veterinary microbiologists. Immunologists and specialists in infectious diseases and national symposium on trends in vaccinology for animal diseases, p. 84 . 